\title{
Towards the QCD phase diagram
}

\section{Philippe de Forcrand*}

Institut für Theoretische Physik, ETH Zürich, CH-8093 Zürich, Switzerland and CERN, Physics Department, TH Unit, CH-1211 Geneva 23, Switzerland

E-mail: forcrandephys.ethz.ch

\section{Owe Philipsen}

Institut für Theoretische Physik, Westfälische Wilhelms-Universität Münster, Germany

E-mail: ophil@uni-muenster.de

\begin{abstract}
We summarize our recent results on the phase diagram of QCD with $N_{f}=2+1$ quark flavors, as a function of temperature $T$ and quark chemical potential $\mu$. Using staggered fermions, lattices with temporal extent $N_{t}=4$, and the exact RHMC algorithm, we first determine the critical line in the quark mass plane $\left(m_{u, d}, m_{s}\right)$ where the finite temperature transition at $\mu=0$ is second order. We confirm that the physical point lies on the crossover side of this line. Our data are consistent with a tricritical point at $\left(m_{u, d}, m_{s}\right)=(0, \sim 500) \mathrm{MeV}$.

Then, using an imaginary chemical potential, we determine in which direction this second-order line moves as the chemical potential is turned on. Contrary to standard expectations, we find that the region of first-order transitions shrinks in the presence of a chemical potential, which is inconsistent with the presence of a QCD critical point at small chemical potential.

The emphasis is put on clarifying the translation of our results from lattice to physical units, and on discussing the apparent contradiction of our findings with earlier lattice studies.
\end{abstract}

XXIVth International Symposium on Lattice Field Theory

July 23-28, 2006

Tucson, Arizona, USA

*Speaker. 


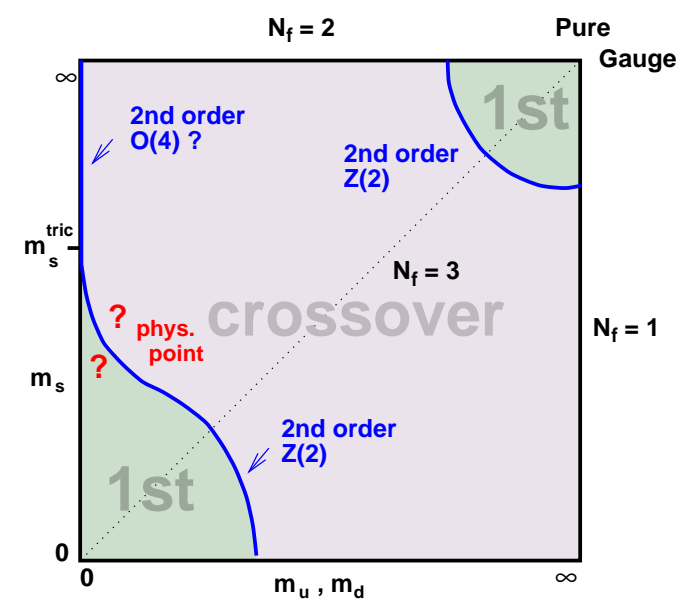

Figure 1: Schematic phase transition behaviour of $N_{f}=2+1$ flavor QCD for different choices of quark masses $\left(m_{u, d}, m_{s}\right)$, at $\mu=0$ (from [1]).

\section{Introduction}

In recent years, considerable efforts have been devoted to the determination of the phase diagram of QCD at finite temperature and density [1]. At zero chemical potential, the behaviour as a function of temperature depends on the quark masses $m_{u, d}$ and $m_{s}$, and expectations are summarized in Fig. 1. In the limits of zero and infinite quark masses (lower left and upper right corners), order parameters corresponding to the breaking of a symmetry can be defined, and one finds numerically that a first-order transition takes place at a finite temperature $T_{c}$. On the other hand, one observes an analytic crossover at intermediate quark masses. Hence, each corner must be surrounded by a region of first-order transition, bounded by a second-order line as in Fig. 1. The line in the heavy-quark corner has been studied in [2]. Here, we want to determine the chiral critical line.

Along both lines, the universality class is that of the $3 d$ Ising model. Therefore, a powerful tool to determine the critical couplings is the Binder cumulant $B_{4} \equiv\left\langle\delta X^{4}\right\rangle /\left\langle\delta X^{2}\right\rangle^{2}$, where $\delta X=$ $X-\langle X\rangle$, and we take for $X$ the $u, d$ quark condensate $\bar{\psi} \psi$. On lattices $8^{3}, 12^{3}$ and $16^{3} \times 4$, we estimate the critical couplings as those for which $B_{4}=1.604 .$. , the $3 d$ Ising value. For each mass point $\left(m_{u, d}, m_{s}\right)$, we accumulate at least 200k RHMC trajectories, and interpolate among 4 or more $m_{u, d}$ values to find the critical $m_{u, d}$ mass $m^{c}$ at a given $m_{s}$. We obtain the set of points in Fig. 4, left.

We then consider the effect of a baryonic chemical potential. As a function of $\mu$, represented vertically in Fig. 2, the critical line determined at $\mu=0$ spans a surface. The standard expectation is depicted in Fig. 2 left. The first order region expands as $\mu$ is turned on, so that the physical point, initially in the crossover region, eventually belongs to the critical surface. At that chemical potential $\mu_{E}$, the transition is second order: that is the QCD critical point. Increasing $\mu$ further makes the transition first order. A completely different scenario arises if instead the first-order region shrinks as $\mu$ is turned on. In that case (Fig. 2 right), the physical point remains in the crossover region for any $\mu$. Since the phenomenologically interesting question is whether a QCD critical point $\left(\mu_{E}, T_{E}\right)$ exists at small $\mu_{E}, \mu_{E} / T_{E} \lesssim 1$, this question can be addressed by an analytic continuation based on a Taylor expansion. Using an imaginary chemical potential $[3,4]$, we determine the curvature 

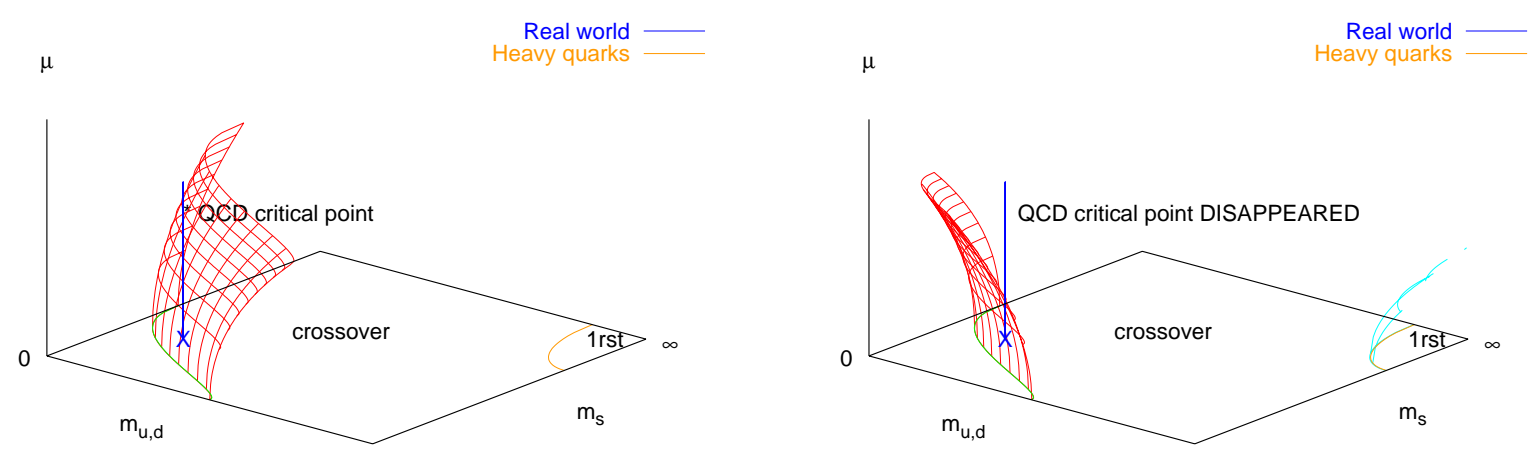

Figure 2: The chiral critical surface in the case of positive (left) and negative (right) curvature. If the physical point is in the crossover region for $\mu=0$, a finite $\mu$ phase transition will only arise in the scenario (left) with positive curvature, where the first-order region expands with $|\mu|$. Note that for heavy quarks, the first-order region shrinks with $|\mu|$ (right) [5].

$\left.\frac{d m^{c}}{d \mu^{2}}\right|_{\mu=0}$ of the critical surface at $\mu=0$. We find that it is negative, so that the first-order region shrinks as in Fig. 2 right. Note that in the opposite corner, the first-order region also shrinks [5].

Sec. 2 tests our methodology in the $N_{f}=3$ case. Sec. 3 describes the $N_{f}=2+1$ study. Sec. 4 compares our results with earlier lattice studies and discusses the various limitations of our approach.

2. $N_{f}=3$

We first check our methodology in the case of 3 degenerate flavors. This is basically a repeat of Ref. [6], this time using the RHMC algorithm [7] instead of the R algorithm.

The RHMC algorithm eliminates the stepsize error of the $\mathrm{R}$ algorithm, which differs in magnitude in the chirally symmetric and broken phases [8]. As a result, the value of $m^{c}(\mu=0)$ is considerably different: $\left(\operatorname{am}^{c}(\mu=0)\right)$ moves from 0.033(1) (R alg.) [6] to 0.0260(5) (see Fig. 3, left). We have checked, by performing zero-temperature simulations at this quark mass, that this is not a simple renormalization effect, but that the physical ratio $m_{\pi} / T_{c}$ is lowered by about $10 \%$. Therefore, an exact algorithm appears mandatory for the study of the $N_{f}=2+1$ critical line. Moreover, RHMC turns out to be vastly more efficient, by up to a factor 20 in our case for the smallest quark masses [9].

We now turn on an imaginary chemical potential $\mu=i \mu_{I}$, and for each $\mu_{I}$ monitor the Binder cumulant $B_{4}$ as a function of the quark mass. Our results are summarized in Fig. 3, left. The chemical potential has almost no influence on $B_{4}$. A lowest-order fit, linear in $a m$ and $(a \mu)^{2}$, gives the error band Fig. 3, right, corresponding to

$$
a m^{c}(a \mu)=0.0270(5)-0.0024(160)(a \mu)^{2}
$$

Care must be taken for the conversion to physical units. The crucial point is that, as we increase the chemical potential $\mu_{I}$, we tune the gauge coupling $\beta$ upwards to maintain criticality, 

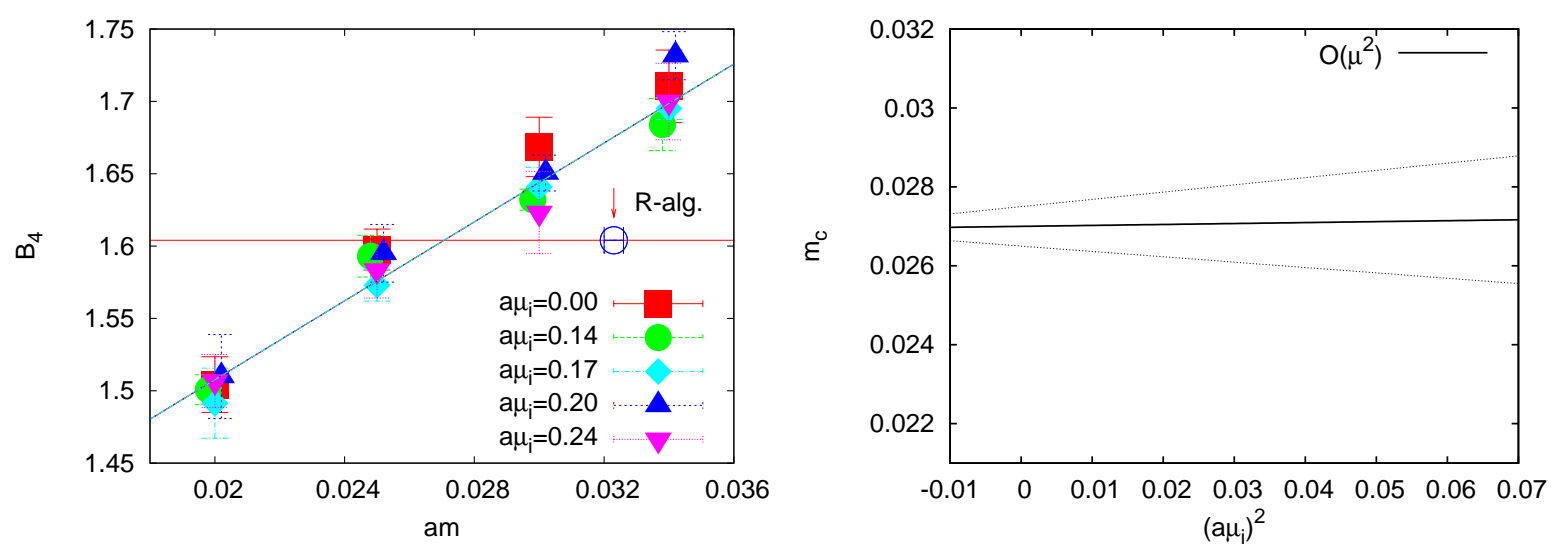

Figure 3: Left: $B_{4}\left(a m, a \mu_{I}\right)$ for different imaginary chemical potentials. Right: One-sigma error band for the critical mass $a m^{c}\left(a \mu_{I}\right)$ resulting from a linear fit.

so that $a(\beta)$ decreases: our observation that $a m^{c}\left(\mu_{I}\right) \approx$ const. does not mean that $m^{c}\left(\mu_{I}\right) \approx$ const., but that $m^{c}\left(\mu_{I}\right)$ increases with $\mu_{I}$, or decreases with a real chemical potential $\mu$. If we express

$$
\begin{gathered}
\frac{a m^{c}(\mu)}{a m^{c}(0)}=1+\frac{c_{1}^{\prime}}{a m^{c}(0)}(a \mu)^{2}+\ldots \\
\frac{m^{c}(\mu)}{m^{c}(0)}=1+c_{1}\left(\frac{\mu}{\pi T}\right)^{2}+\ldots
\end{gathered}
$$

then $c_{1}$ and $c_{1}^{\prime}$ are related by

$$
c_{1}=\frac{\pi^{2}}{N_{t}^{2}} \frac{c_{1}^{\prime}}{a m^{c}(0)}+\left(\frac{1}{T_{c}(m, \mu)} \frac{d T_{c}(m, \mu)}{d(\mu / \pi T)^{2}}\right)_{\mu=0}
$$

where $m=m^{c}(\mu)$ in the second term. Writing the transition temperature as

$$
\frac{T_{c}(m, \mu)}{T_{c}\left(m_{0}^{c}, 0\right)}=1+A \frac{m-m_{0}^{c}}{\pi T}+B\left(\frac{\mu}{\pi T}\right)^{2}+\ldots
$$

one obtains

$$
c_{1}=\left(B+\frac{\pi^{2}}{N_{t}^{2}} \frac{c_{1}^{\prime}}{a m^{c}(0)}\right)\left(1-A \frac{m_{0}^{c}}{\pi T}\right)^{-1} .
$$

$c_{1}^{\prime}$ and $\frac{m_{0}^{c}}{\pi T}$ are both small, so that $c_{1}$ is nearly equal to $B$. Estimates of $B$ and $A$ can be obtained by converting our result for the pseudo-critical gauge coupling

$$
\beta_{0}(a m, a \mu)=5.1369(3)+1.94(3)\left(a m-a m_{0}^{c}\right)+0.781(7)(a \mu)^{2}
$$

to physical units. Using the 2-loop $\beta$-function gives $A=2.111(17), B=-0.667(6)$ so that finally

$$
\frac{m^{c}(\mu)}{m^{c}(0)}=1-0.7(4)\left(\frac{\mu}{\pi T}\right)^{2}+\ldots
$$

The error above is conservative and includes the uncertainty from using different fitting forms (see Table 2, Ref. [10]). The main source of systematic error comes from using the 2-loop $\beta$-function 

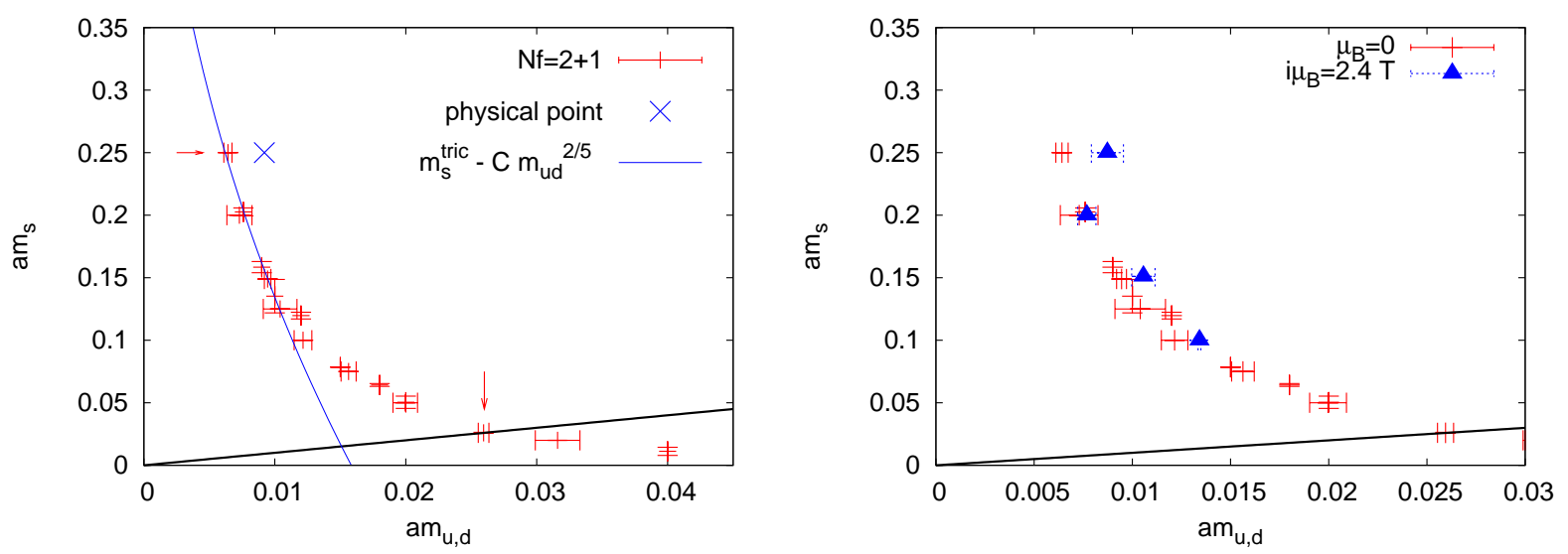

Figure 4: Left: The chiral critical line in the bare quark mass plane at $\mu=0 . N_{f}=3$ is shown by the solid line. Also shown are the physical point according to [12], and a fit corresponding to a tricritical point $m_{s}^{\text {tric }} \sim 2.8 T$. Right: Comparison of the critical line at $\mu=0$ and $a \mu_{I}=0.2$.

to obtain $B$. The non-perturbative $\beta$-function varies more steeply and may increase $A$ and $B$, in magnitude, by up to a factor 2 . This will make $c_{1}$ more negative.

We thus have clear evidence that, in the $N_{f}=3$ theory on an $N_{t}=4$ lattice, the region of firstorder transitions shrinks as a baryon chemical potential is turned on, and the "exotic scenario" of Fig. 2, right, is the correct one. This result is further supported by recent simulations of the same theory, under an isospin chemical potential [11].

\section{3. $N_{f}=2+1$}

We now proceed to the non-degenerate case. First, at $\mu=0$, we map out the line of secondorder transitions in the $\left(a m_{u, d}, a m_{s}\right)$ plane. Our results, shown Fig. 4, left, are in qualitative agreement with expectations Fig. 1. In particular, they are consistent with the possible existence of a tricritical point $\left(m_{u, d}=0, m_{s}=m_{s}^{\text {tric }}\right)$. Using its known, Gaussian exponents, our data favor (blue line in Fig. 4 left) a heavy $m_{s}^{\text {tric }} \sim 2.8 T_{c}$.

A more immediate issue is whether the QCD physical point lies on the crossover side of the critical line as expected. For that purpose, we have performed spectrum calculations at $T \sim 0$, at the parameters corresponding to the horizontal arrow in Fig. 4 left $\left(a m_{u, d}=0.005, a m_{s}=0.25, \beta=\right.$ 5.1857). They show that $m_{s}$ is approximately tuned to its physical value $\left(\left.\frac{m_{K}}{m_{\rho}} \sim \frac{m_{K}}{m_{\rho}}\right|_{\text {phys }}\right)$, while the pion is lighter than in QCD $\left(\frac{m_{\pi}}{m_{\rho}}=0.148(2)<0.18\right)$. This confirms that the physical point lies on the right of the critical line, i.e. in the crossover region ${ }^{1}$. This conclusion has been confirmed by very recent calculations on finer lattices [13]. Also, we find $T_{c}$ to vary little along the critical line, in accordance with model calculations [14].

We now couple an imaginary chemical potential $a \mu_{I}=0.2$ to the two light flavors, and measure the change in the critical mass $a m_{u, d}$ as in the $N_{f}=3$ case. Fig. 4 right shows the same trend as for $N_{f}=3$ : the critical mass is constant or slightly increasing, in lattice units. The conversion to

\footnotetext{
${ }^{1}$ In fact, our estimate of the lattice parameters corresponding to the physical point is consistent with that of Fodor \& Katz using the same action, but the R algorithm [12].
} 
physical units proceeds as in eqs.(2.2-2.8). Since the critical gauge coupling $\beta_{0}\left(a \mu_{I}\right)$ increases with $\mu_{I}$, the coefficient $B$, which is the dominant contribution to $c_{1}$, is negative. Together with a very small or slightly negative value for $c_{1}^{\prime}$, it implies again that the first-order region shrinks as the baryon chemical potential is turned on, and the "exotic scenario" of Fig. 2, right, is the correct one.

This statement comes with several caveats: $(i)$ our lattice is very coarse $(a \sim 0.3 \mathrm{fm})$; $(i i)$ as we consider lighter $m_{u, d}$, our box becomes small ( $m_{\pi} L \sim 1.7$ for the worst case); (iii) we use "rooting" of the staggered determinant to simulate 1 and 2 flavors, albeit our measure is positive with an imaginary $\mu$, so that we avoid the pitfalls of [15].

\section{Discussion}

Our results appear in qualitative contradiction with those of Fodor \& Katz [12] and of Gavai $\&$ Gupta [16], which both conclude for the existence of a critical point $\left(\mu_{E}, T_{E}\right)$ at small chemical potential $\mu_{E} / T_{E} \lesssim 1$. Let us consider the reasons for such disagreement.
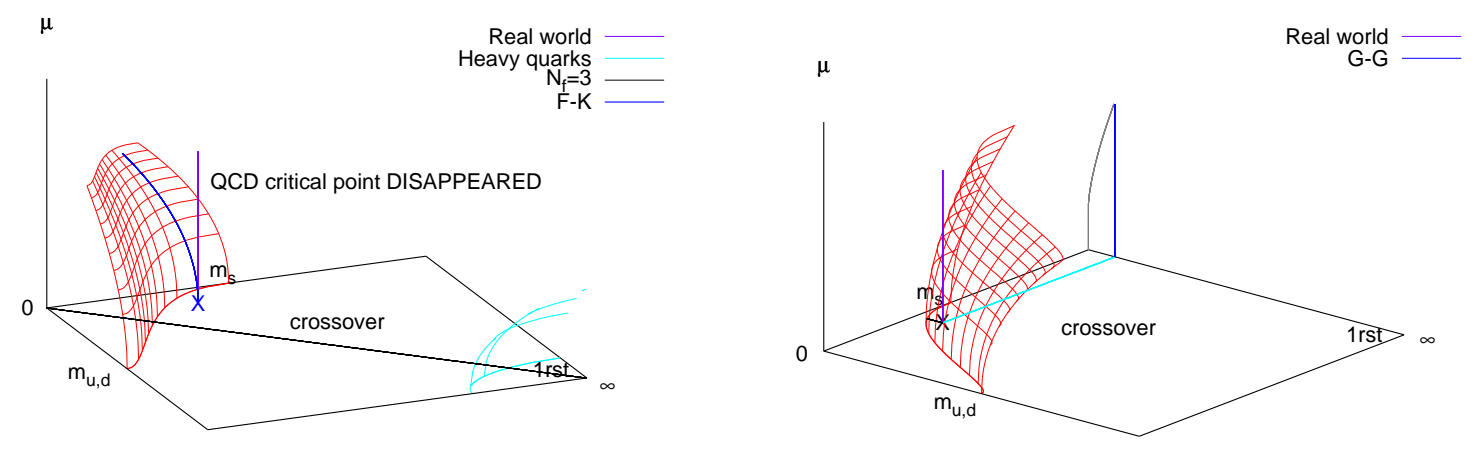

Figure 5: Left: Effect of keeping the quark mass fixed in lattice units in [12]. Right: Comparison at finite $\mu$ between the $N_{f}=2+1$ and the $N_{f}=2$ theory considered in [16].

- Fodor \& Katz obtain Monte Carlo results at $\beta=\beta_{c}, \mu=0$, and perform a double reweighting in $(\beta, \mu)$ along the pseudo-critical line $\beta_{c}(a \mu)$. By construction, this reweighting is performed at a quark mass fixed in lattice units: $a m_{u, d}=\frac{m_{u, d}}{T_{c}}=$ const.. Since the critical temperature $T_{c}$ decreases as they turn on $\mu$, so does their quark mass. This decrease of the quark mass pushes the transition towards first order, which might be the reason why they find a critical point at small $\mu$. This effect is illustrated in the sketch Fig. 5, left, where the bent trajectory à la Fodor \& Katz intersects the critical surface, while the vertical line of constant physics does not.

Put another way, Fodor \& Katz measure the analogue of eq.(2.2) instead of (2.3). From their Fig. 1 (Ref. [12]), the coefficient $c_{1}^{\prime}$ which one would extract would be essentially zero like ours. As in our case, the variation of $T_{c}$ with $\mu$ makes a dominant contribution, which may change the results qualitatively.

- Gavai \& Gupta try to infer the location of the critical point by estimating the radius of convergence of the Taylor expansion of the free energy in $(\mu / \pi T)^{2}$. Regardless of the systematic error attached to such estimate when only 4 Taylor coefficients are available, we want to point out 
that they consider a theory without strange quark, i.e. $N_{f}=2$ only. The $(\mu, T)$ phase diagram of such a theory is qualitatively different from that of $N_{f}=2+1$ QCD. At $\mu=0$, the order of the finite-temperature transition as $m_{u, d} \rightarrow 0$ is not settled [17]. Assuming a second-order $O(4)$ transition, one expects then a tricritical point at $\left(m_{u, d}=0, \mu=\mu^{\text {tric }}\right)$, beyond which a non-zero critical mass $m_{u, d}^{c}(\mu)$ can be defined, as sketched in Fig. 5 right. The quantitative relevance of results, even accurate, for this $N_{f}=2$ theory to QCD is unclear to us.

Therefore, we find no inconsistency between our results and those above. We conclude that the existence of a critical point $\left(\mu_{E}, T_{E}\right)$ in QCD at small chemical potential $\mu_{E} / T_{E} \lesssim 1$ is an open question. Our numerical evidence, with the caveats mentioned in Sec. 3, is that the curvature of the critical surface is as illustrated Fig. 2 right. Our main systematic error comes from our coarse lattice spacing $a \sim 0.3 \mathrm{fm}$ [18]. If confirmed on a finer lattice, the implications of our finding are as follows. In the region where a leading Taylor expansion of the critical surface is a good approximation, i.e. $\mu / T \lesssim 1$, corresponding to the experimentally accessible regime, no critical point exists which is analytically connected to $\mu=0$. Of course, we cannot exclude that the QCD phase diagram is more complex, and partly inaccessible to our imaginary $\mu+$ Taylor expansion strategy.

\section{References}

[1] E. Laermann and O. Philipsen, Ann. Rev. Nucl. Part. Sci. 53 (2003) 163 [arXiv:hep-ph/0303042]; O. Philipsen, PoS LAT2005 (2006) 016 [PoS JHW2005 (2006) 012 [arXiv:hep-lat/05100677.

[2] P. Hasenfratz, F. Karsch and I. O. Stamatescu, Phys. Lett. B 133 (1983) 221; C. Alexandrou et al., Phys. Rev. D 60 (1999) 034504 [arXiv:hep-lat/9811028]; A. Dumitru, D. Roder and J. Ruppert, Phys. Rev. D 70 (2004) 074001 [arXiv:hep-ph/0311119].

[3] P. de Forcrand and O. Philipsen, Nucl. Phys. B 642 (2002) 290 [arXiv:hep-lat/0205016].

[4] M. D’Elia and M. P. Lombardo, Phys. Rev. D 67 (2003) 014505 [arXiv:hep-lat/0209146].

[5] S. Kim et al., PoS LAT2005 (2006) 166 [arXiv:hep-lat/0510069].

[6] P. de Forcrand and O. Philipsen, Nucl. Phys. B 673 (2003) 170 [arXiv:hep-lat/0307020].

[7] M. A. Clark et al., Nucl. Phys. Proc. Suppl. 140 (2005) 835 [arXiv:hep-lat/0409133].

[8] J. B. Kogut and D. K. Sinclair, arXiv:hep-lat/0504003.

[9] M. A. Clark et al., PoS LAT2005 (2006) 115 [arXiv:hep-lat/0510004].

[10] P. de Forcrand and O. Philipsen, arXiv:hep-lat/0607017.

[11] D. K. Sinclair and J. B. Kogut, arXiv:hep-lat/0609041.

[12] Z. Fodor and S. D. Katz, JHEP 0404 (2004) 050 [arXiv:hep-lat/0402006].

[13] Y. Aoki, G. Endrodi, Z. Fodor, S.D. Katz and K.K. Szabo, Nature 443, 675 - 678 (12 October 2006).

[14] Z. Szep, PoS JHW2005 (2006) 017 [arXiv:hep-ph/0512241].

[15] M. Golterman, Y. Shamir and B. Svetitsky, Phys. Rev. D 74 (2006) 071501 [arXiv:hep-lat/0602026].

[16] R. V. Gavai and S. Gupta, Phys. Rev. D 71 (2005) 114014 [arXiv:hep-lat/0412035].

[17] J. B. Kogut and D. K. Sinclair, Phys. Rev. D 73 (2006) 074512 [arXiv:hep-lat/0603021]; M. D’Elia, A. Di Giacomo and C. Pica, Phys. Rev. D 72 (2005) 114510 [arXiv:hep-lat/0503030].

[18] F. Karsch et al., Nucl. Phys. Proc. Suppl. 129 (2004) 614 [arXiv:hep-lat/0309116]. 